\title{
An Error Bounded Tangent Estimator for Digitized Elliptic Curves
}

\author{
Dilip K. Prasad, Raj Kumar Gupta, and Maylor K.H. Leung \\ School of Computer Engineering, Nanyang Technological University, Singapore \\ dilipprasad@gmail.com
}

\begin{abstract}
In this paper, we address the problem of tangent estimation for digital curves. We propose a simple, geometry based tangent estimation method for digital curves. The geometrical analysis of the method and the maximum error analysis for digital elliptic curves are presented. Numerical results have been tested for digital ellipses of various eccentricities (circle to very sharp ellipses) and the maximum error of the proposed method is bounded and is less than 5.5 degrees for reasonably large ellipses. The error for digital circles is also analyzed and compared with a recent tangent estimation method. In addition, the tangent estimation technique is applied to a flower shaped digital curve with six inflexion points and the results demonstrate good performance. The proposed tangent estimator is applied to a practical application which analyzes the error in a geometric ellipse detection method. The ellipse detection method is greatly benefited by the proposed tangent estimator, as the maximum error in geometrical ellipse detection is no more critically dependent upon the tangent estimation (due to the reduced error in tangent estimation). The proposed tangent estimator also increases the reliability and precision of the ellipse detection method.
\end{abstract}

Keywords: Tangent estimation, digital curves, error analysis, elliptic curves.

\section{Introduction}

Many image processing applications require tangent estimation for digital curves [1-10]. Examples include corner detection, inflection point detection, shape representation, Hough transform based methods for conic fitting, projective estimation of 3-dimensional shapes.

Geometric problems like estimating tangents, curvature, or shape features are well established for continuous non-digitized parametric curves. These problems become significantly difficult in the digitized pixel space of images, as the analytical equations may not take any continuous solution. The chosen solution is almost always an approximate integer solution nearest to the actual solution of the analytic equations. On one hand, digitization severely reduces the information contained in the continuous curves. On the other hand, the continuous curve can be analytically characterized using equation of finite number of coefficients (geometric parameters). Digitization introduces a non-linear corruption in the continuous curve which cannot be analyzed using equations [8-12]. 
Estimating the tangents in digitized curves is challenging because of three main reasons:

- The tangent is defined typically on a point, though it is a property of the continuous curve to which the point belongs. Thus, it has the local as well as the global properties of the curve. Due to the digitization, both these properties are affected and the nature or extent of effect cannot be quantified or analyzed using simple mathematical tools. At best, some estimates of maximum error or localized precision may be developed.

- Usually, while estimating the tangents, prior information about the nature of the curve is not available. Further, appropriate size of the local region around a point is also not known. Choosing these parameters is mainly heuristic based and not robust.

- Digitization permits many similar (though not the same) curves to be fit on the same digital pixel sequence. Thus, the tangents can never be obtained uniquely for the digital curves.

There are several approaches for tangent estimation in the case of digital curves. One of the methods to find the tangents is to use continuous function (typically second order) to approximate the curvature of the digital curve in a local region around the point of interest [13-14]. Then the derivative of the continuous function is used to determine the tangent. Such approach is restrictive in the choice of the nature of continuous function and the definition, shape, and dimension of the local region, etc. Besides being computationally intensive, they are also afflicted by the quantization noise. Further, there are applications where tangents need to be computed to fit a shape (for example ellipse) on the digital curve. In such cases, it is difficult to rely on a method that first fits a shape in the local region to estimate the tangent, and then uses the tangent to fit a shape to the whole curve. In order to overcome the problem of choosing the continuous function, researchers sometimes use a Gaussian filter to smoothen the digital curve and obtain a smooth continuous curve. This Gaussian smoothened continuous curve is then used for estimating the tangents [15].

Yet another method is to consider a family of continuous curves of various types. The whole digital curve is approximated by one of the continuous curves in the family using a global optimization technique. Then the tangents are computed on the curve chosen by optimization [16].

A different approach is to approximate the digital curves using line segments. Two main variations in this approach are in vogue. The first variation is based on the maximal segments [8, 11-12]. At the point of interest, the maximal line segments passing through it are found and weighted convex combination of their slopes is used to find the orientation of the tangent. Though this method is parameter-free, has asymptotic convergence, and incorporates convexity property, it is developed basically on heuristics, rather than analytic foundation.

Another variation is to approximate the digital curve using small line segments such that the maximum deviation of any point on the digital curve with one of the fitted line segments is small, below a threshold value of a few pixels [17]. This procedure divides the curve into small sub-curves each corresponding to a fitted line segment. Then the slope of the tangent at the midpoint of each sub-curve is 
considered to be the same as the slope of the corresponding line segment. The main restriction with this method is that the tangents are available only at some points of the digital curves, viz., the mid points of the digital sub-curves.

We propose a tangent estimation method that is considerably simpler than all the above methods and has firm analytical foundation. Further, for estimating the slope of the tangent, only two points at a certain distance from the point of interest need to be found and no apriori information about the nature of curve is required. We prove that in the case of non-digitized ellipses, the slope computed by our method matches the slope of the actual tangent at any point on the curve. Though the proof is presented for elliptic curve only, it can be extended to all the conics. We considered elliptic curve because of its wide applicability. For the case of digital curve, we derive the expressions of the maximum error in tangent estimation. Based on the derivation, we compute the maximum error in the tangent estimation for a large range of ellipses, which include small circles to highly eccentric ellipses. The results sufficiently demonstrate the strength of the proposed tangent estimation method.

We also consider an example where this analysis of the upper bound of the error has significant influence. In the three point geometric Hough transform method [7], it was shown that the error in tangent estimation is the most important practical contributor of the error [1]. It was also shown that if the maximum error in computation is known, a reliability region for the computed centers can be predicted based upon a probability density function [1]. We use the maximum error for the proposed tangent estimation for this application.

In the following, Section 2 presents the proposed tangent estimation method and the geometrical proof of the concept. Section 3 analyzes the maximum error in the tangent estimation for digital curves using the proposed method. Section 4 presents numerical examples to illustrate the effectiveness of the proposed tangent estimator. Section 5 shows an application of the proposed tangent estimation method for ellipse detection. Section 6 concludes the article.

\section{Proposed Tangent Estimation Method}

In this section, we present the proposed tangent estimation method. The discussion has been restricted to elliptic curves. The analysis is easily extensible to any other conic as well. This section develops and tests the concept for the continuous curve only. We introduce the concept in section 2.1 and present the geometric proof of the validity of the concept in section 2.2 .

\subsection{The Concept}

Let us consider an ellipse:

$$
(x / a)^{2}+(y / b)^{2}=1
$$

where, $a$ and $b(a \geq b)$ are the lengths of semi-major and semi-minor axes. Suppose we are interested in finding the tangent at the point $P_{0}\left(x_{0}, y_{0}\right)$. We know that the actual slope of the tangent at $P_{0}$ is given as follows: 


$$
m_{0}=\frac{\mathrm{d} y}{\mathrm{~d} x}=-\left(\frac{b}{a}\right)^{2} \frac{x_{0}}{y_{0}}
$$

In reality, since we do not know the curve to which $P_{0}$ belongs, we cannot compute the tangent analytically as above. We propose to use a small circle of radius $R \ll b$ centered at $P_{0}$ :

$$
\left(x-x_{0}\right)^{2}+\left(y-y_{0}\right)^{2}=R^{2}
$$

The circle intersects the ellipse given by (1) at points $P_{1}$ and $P_{2}$. There are two steps for finding the tangent at $P_{0}$. First, find the slope of the line $P_{1} P_{2}$ (denoted by $\tilde{m}$ ). Second, find a line with slope $\tilde{m}$ passing through the point $P_{0}$.

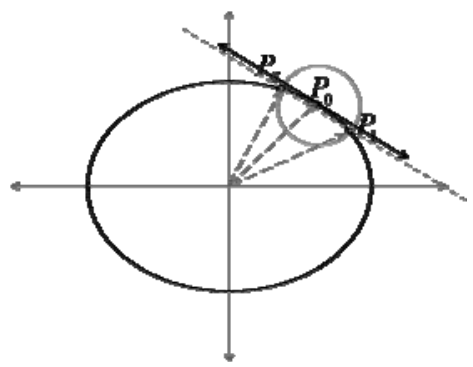

Fig. 1. Illustration of the geometric concept used for tangent estimation at the point $P_{0}$

The idea is demonstrated in Figure 1 above. The slope $\tilde{m}$ of the line $P_{1} P_{2}$ is given by:

$$
\tilde{m}=\left(y_{2}-y_{1}\right) /\left(x_{2}-x_{1}\right)
$$

\subsection{Geometric Proof of the Proposed Concept}

For analysing the error in the proposed method we first need to find the coordinates of points $P_{1}$ and $P_{2}$. For the ease of analysis, we define the coordinates of the points on the ellipse using a parametric notation as:

$$
x=a \cos \theta ; \quad y=b \sin \theta
$$

Specifically, the coordinates of $P_{0}$ are given by $\left(a \cos \theta_{0}, b \sin \theta_{0}\right)$. It should be noted that $\theta$ is not a geometric angle. Then, we can substitute (5) and the coordinates of $P_{0}$ in (3) to find the intersection points $P_{1}$ and $P_{2}$ :

$$
\left(a \cos \theta-a \cos \theta_{0}\right)^{2}+\left(b \sin \theta-b \sin \theta_{0}\right)^{2}=R^{2}
$$

The above equation can be simplified using algebraic and trigonometric manipulations as follows: 


$$
4 \sin ^{2}\left(\frac{\theta-\theta_{0}}{2}\right)\left(a^{2} \sin ^{2}\left(\frac{\theta+\theta_{0}}{2}\right)+b^{2} \cos ^{2}\left(\frac{\theta+\theta_{0}}{2}\right)\right)=R^{2}
$$

The solutions of the above equation gives the intersection points. The derivation for solving the above equation is very long and tedious. For brevity, we circumvent the derivation and state that for $R \ll b$, the value of $\theta$ is close to $\theta_{0}$ :

$$
\theta=\theta_{0}+\Delta \theta ; \quad \Delta \theta \approx 0
$$

Then (7) can be written as:

$$
\begin{gathered}
\lim _{\Delta \theta \rightarrow 0}\left\{4 \sin ^{2}\left(\frac{\theta-\theta_{0}}{2}\right)\left(a^{2} \sin ^{2}\left(\frac{\theta+\theta_{0}}{2}\right)+b^{2} \cos ^{2}\left(\frac{\theta+\theta_{0}}{2}\right)\right)\right. \\
=4 \sin ^{2}\left(\frac{\Delta \theta}{2}\right)\left(a^{2} \sin ^{2} \theta_{0}+b^{2} \cos ^{2} \theta_{0}\right)=R^{2}
\end{gathered}
$$

Thus, $\Delta \theta$ can be computed as follows:

$$
\Delta \theta= \pm 2 \sin ^{-1}\left(\sqrt{\frac{R^{2}}{4\left(a^{2} \sin ^{2} \theta_{0}+b^{2} \cos ^{2} \theta_{0}\right)}}\right)
$$

Then the coordinates of $P_{1}$ correspond to the negative value of $\Delta \theta$, while the coordinates of $P_{2}$ correspond to the positive value of $\Delta \theta$, i.e. $\theta_{2}+\theta_{1}=2 \theta_{0}$, and the slope of the line $P_{1} P_{2}$ is given as follows:

$$
\tilde{m}=\frac{b \sin \theta_{2}-b \sin \theta_{1}}{a \cos \theta_{2}-a \cos \theta_{1}}=-\frac{b}{a} \cot \left(\frac{\theta_{2}+\theta_{1}}{2}\right)=-\frac{b}{a} \cot \left(\theta_{0}\right)
$$

Substituting the parametric coordinates for $x_{0}$ and $y_{0}$ in (2), we get:

$$
m_{0}=\frac{\mathrm{d} y}{\mathrm{~d} x}=-\frac{b}{a} \cot \theta_{0}
$$

We notice from (11) and (12) that in the analytical case (absence of digitization), if $R \ll b$, then there is no error in the computation of the slope with the proposed method.

\subsection{Choice of $R$}

The aim in choosing $R \ll b$ is that $\Delta \theta \approx 0$ and consequently $\sin (\Delta \theta / 2) \approx 0$, such that (9) is valid. Suppose we choose a maximum value of $\Delta \theta$, denoted by $\Delta \theta_{\max }$, then using (10) and $\theta_{0}=0$, the expression for choosing $R$ is given as:

$$
R \leq 2 b \sin \left(\Delta \theta_{\max } / 2\right)
$$

For example, if we use $\Delta \theta_{\max }=(\pi / 18)$, i.e., $10^{\circ}$, such that $\sin (\Delta \theta / 2)=0.0872$, then $R \leq 0.1743 b$. 


\section{Maximum Error in Tangent Estimation Due to Digitization}

Due to digitization in the case of images, a general point $P(x, y)$ is approximated by a pixel $P^{\prime}\left(x^{\prime}, y^{\prime}\right)$ as follows:

$$
x^{\prime}=\operatorname{round}(x) ; \quad y^{\prime}=\operatorname{round}(y)
$$

where round $(x)$ denotes the rounding the value of real number $x$ to its nearest integer. $P^{\prime}\left(x^{\prime}, y^{\prime}\right)$ satisfy the following:

$$
\begin{gathered}
x^{\prime}, y^{\prime} \in \mathbb{Z} \\
x^{\prime}=x+\Delta x ; \quad y^{\prime}=y+\Delta y \\
-0.5 \leq \Delta x \leq 0.5, \quad-0.5 \leq \Delta y \leq 0.5
\end{gathered}
$$

We shall use (16) and (17) for estimating the maximum error in the computation of the slope of the numeric tangent $\tilde{m}$. Let the slope of numeric tangent computed by pixels $P_{1}^{\prime}\left(x_{1}^{\prime}, y_{1}^{\prime}\right)$ and $P_{2}^{\prime}\left(x_{2}^{\prime}, y_{2}^{\prime}\right)$ (corresponding to $P_{1}$ and $\left.P_{2}\right)$ be denoted by $\tilde{m}^{\prime}$. We shall call the numeric tangent computed with pixels as the digital tangent. Then $\tilde{m}^{\prime}$ can be solved as follows:

$$
\tilde{m}^{\prime}=\frac{y_{2}^{\prime}-y_{1}^{\prime}}{x_{2}^{\prime}-x_{1}^{\prime}}=\left(\tilde{m}+\frac{\Delta y_{2}-\Delta y_{1}}{x_{2}-x_{1}}\right) /\left(1+\frac{\Delta x_{2}-\Delta x_{1}}{x_{2}-x_{1}}\right)
$$

The angular difference between the numeric tangent and the digital tangent is used as the estimate of the error. This angular difference is given as:

$$
\partial \phi=\tan ^{-1}(\tilde{m})-\tan ^{-1}\left(\tilde{m}^{\prime}\right)=\tan ^{-1}\left(\frac{\tilde{m}-\tilde{m}^{\prime}}{1+\tilde{m} \tilde{m}^{\prime}}\right)
$$

Substituting (18) in (19), we get:

$$
\partial \phi=\tan ^{-1}\left(\frac{\tilde{m}\left(\Delta x_{2}-\Delta x_{1}\right)-\left(\Delta y_{2}-\Delta y_{1}\right)}{\left(1+\tilde{m}^{2}\right)\left(x_{2}-x_{1}\right)+\left(\Delta x_{2}-\Delta x_{1}\right)+\tilde{m}\left(\Delta y_{2}-\Delta y_{1}\right)}\right)
$$

Now based on the minimum and maximum possible values of $\Delta x_{1}^{\prime}, \Delta x_{2}^{\prime}, \Delta y_{1}^{\prime}$, and $\Delta y_{2}^{\prime}$, we have the nine cases, corresponding to $\left(\Delta x_{2}-\Delta x_{1}\right) \in\{-1,0,1\}$ and $\left(\Delta y_{2}-\Delta y_{1}\right) \in\{-1,0,1\}$.

However, it can be shown that the maximum error occurs in cases $\left|\Delta x_{2}-\Delta x_{1}\right|=\left|\Delta y_{2}-\Delta y_{1}\right|=1$ :

$$
\partial \tilde{\phi}_{\max }=\max \left(\frac{1}{s^{3}}(\sin \tilde{\phi} \pm \cos \tilde{\phi})\left(s^{2}-s( \pm \cos \tilde{\phi} \pm \sin \tilde{\phi})+( \pm \cos \tilde{\phi} \pm \sin \tilde{\phi})^{2}\right)\right)
$$

where, $s=\sqrt{\left(x_{2}-x_{1}\right)^{2}+\left(y_{2}-y_{1}\right)^{2}}$ and $\tilde{\phi}=\tan ^{-1}(\tilde{m})$. It should be noted that the above derivation of the error bound is applicable to any general continuous line 
connecting any points $P_{1}$ and $P_{2}$ and the corresponding digital line with the slope $\tilde{m}^{\prime}$. However, in the present case, $P_{1}$ and $P_{2}$ are given by (10). Thus, the error bound $\partial \tilde{\phi}_{\max }$ is related to $R$ through $s$.

\section{Numerical Results}

In this section, we present numerical results for the maximum error due to the proposed tangent estimation method. Let us consider that the digital ellipses of interest have the length of semi-minor axis $b \geq 30$. Then, using section 2.3, a reasonable value of $R$ is $R \leq 5.229$.

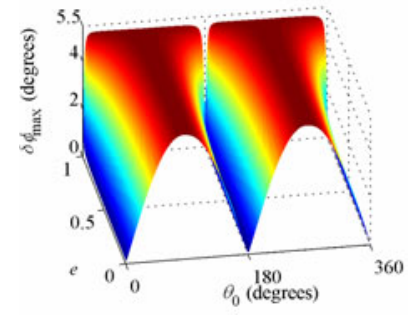

(a) $\partial \phi_{\max }\left(\theta_{0}\right)$ for all various values of eccentricity $e$ and $\theta_{0}$, using $R=3$

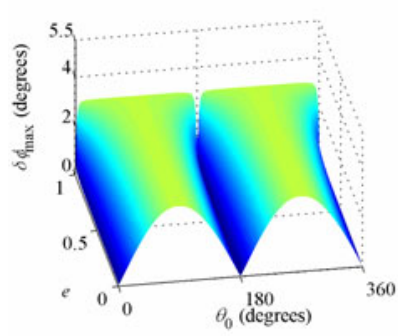

(b) $\partial \phi_{\max }\left(\theta_{0}\right)$ for all various values of eccentricity $e$ and $\theta_{0}$, using $R=5$

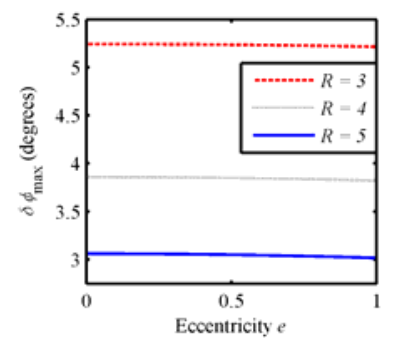

(c) $\max \left(\partial \phi_{\max }\left(\theta_{0}\right) ; \forall \theta_{0}\right)$ for various values of $e$, using $R=3,4,5$

Fig. 2. Numerical results: maximum error in tangent estimation of digital ellipses using $b=30$

We perform three simulations, each with a different value of $R$, viz., $R=3$ and $R=5$ respectively. For each simulation we vary the eccentricity $e=\sqrt{\left(a^{2}-b^{2}\right) / a^{2}}$ of the digital ellipse varies from 0 to 0.999 (circle to extremely sharp ellipses), while keeping $b=30$, and compute $\partial \phi_{\max }\left(\theta_{0}\right)$. The result are presented in Figure $2(\mathrm{a}-\mathrm{b})$. In Figure 2(c), we present $\max \left(\partial \phi_{\max }\left(\theta_{0}\right) ; \forall \theta_{0}\right)$ for various values of eccentricities for $R \in\{3,4,5\}$. It is notable that the maximum error in the computation of the tangents is very small. It is less than $5.25^{\circ}$ for $R=3$ and less than $3.1^{\circ}$ for $R=5$.

Next, we consider digital circles whose radii range from 30 pixels to 10000 pixels. We use three different values of $R$, viz., $R=3, R=4$ and $R=5$ respectively, and compute $\max \left(\partial \phi_{\max }\left(\theta_{0}\right) ; \forall \theta_{0}\right)$. The results shown in Figure 3(a).

In order to understand the difference between the observed maximum error for various values of $R$, let us consider the ratio of the maximum error due to digitization (which is equal to 0.5 ) to the radius $R$ of the circle used for computing the tangent: 
$(0.5 / R)$. It is evident that this ratio is higher for $R=3$ as compared to $R=5$. Thus, the error in the computation of tangent using $R=5$ is less than when $R=3$ is used. If the minimum value of $b$ is higher, such that we can choose larger value of $R$ (satisfying (13)), the maximum error in the computation of tangents will further reduce.

For comparison with the recently proposed $\lambda$-MST estimator, we consider the example test proposed in [12]. Hundred experiments were performed, in each of which a digitized curve corresponding to radius 100 and a randomly chosen center within 1 pixel region were formed and absolute error in the computation of tangent was computed using $\lambda$-MST and the proposed method. The average error in the computation of tangent is shown in Figure 3(b). The results show that the proposed tangent estimation method has lower error than $\lambda$-MST estimator. Further, while the error in the $\lambda$-MST estimator is high at the shift of every quadrant, this feature is not strong in the proposed tangent estimation method.

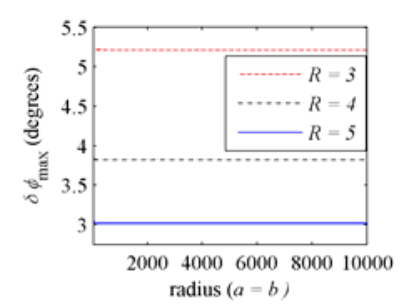

(a) Maximum error in tangent estimation of digital circles with radius from $[30,10000]$ using different values of $R$

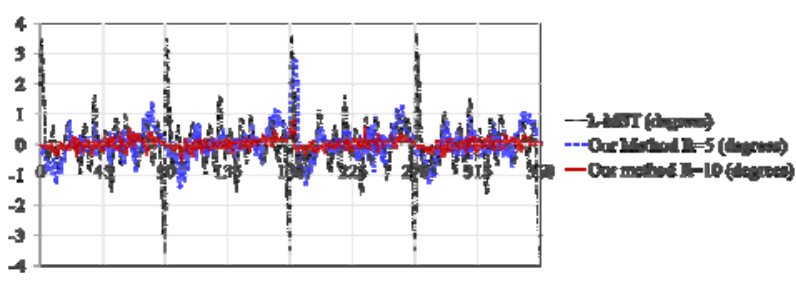

(b) Average absolute error in the computation of tangents for 100 experiments with digitized circles of radius 100 and centers within 1 pixel region chosen randomly. The result is compared with $\lambda$-MST estimator [12]

Fig. 3. Analysis of error for digitized circles

Finally, we show the error in tangent estimation for an analytical shape with inflexion points. We consider the flower shape with 6 petals:

$$
x=\frac{400}{3}(1-1.5 \sin 6 \theta) \cos \theta+60 ; \quad y=\frac{400}{3}(1-1.5 \sin 6 \theta) \sin \theta+60
$$

The digitized shape is shown in Figure 4(a). The smallest circle enclosing this shape completely has a radius 200 . The directions of the tangents on the actual and tangents computed on the digitized curve using $R=20$ are shown in Figure 4(b). The plot shows a good agreement between the actual and computed tangents. The maximum and average errors for various values of $R$ are plotted in Figure 4(c) We mention here that the maximum error occurs when the point of interest is close to the inflexion point. The error at the inflexion point itself is small (close to the average error) due to symmetry of the shape. 


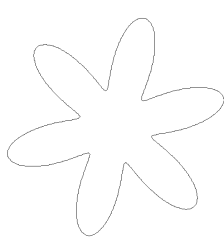

(a) The digitized flower shape represented by (22)

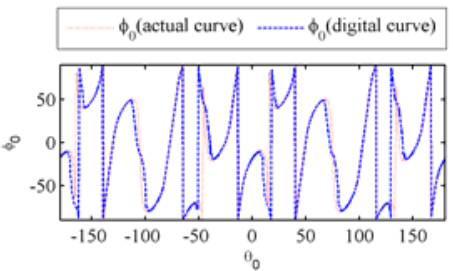

(b) The angle of the tangents on the actual curve and the digital curve (using $R=20$ )

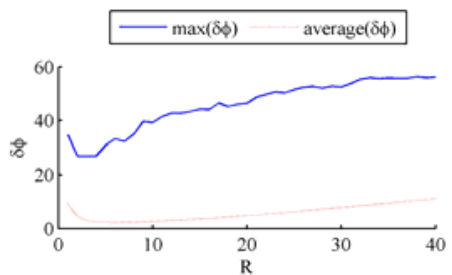

(c) The error in the computation of the tangent due to digitization for various values of $R$

Fig. 4. Example of an analytical curve with inflexion points

\section{Practical Application: Hough Transform Based Ellipse Detection Method (Yuen [7])}

Yuen proposed a variation of randomized Hough transform for detecting ellipses that uses three points on a digital curve to first determine the center of the ellipse and then perform Hough transform [7]. This method was analyzed in [1] and it was shown that the error in the estimation of tangents is the most important contributing factor in this method. In the paper, the result were shown using $\partial \phi_{\max }=15^{\circ}$, which was chosen empirically and is reasonable for the existing methods. However, for the proposed method, we see that the maximum error is less than $5.25^{\circ}$. Thus, the error contributed in Yuen's method [7] for digital ellipses due to the tangent estimation is expected to decrease significantly.

In [1], among the four tests presented for analyzing the relative error $r_{\text {err }} / a$ of ellipse fitting using Yuen's method [7], Test 4(a,b) present the error contributed due to the erroneous tangent estimation. We substitute $\partial \phi_{\max }=5.25^{\circ}$ and compute the results of Test $4(\mathrm{a}, \mathrm{b})$ of [1] considering digitization. The results are presented in Figure 5. It can be seen that the error due to tangents is significantly less than the results presented in [1], which considered $\partial \phi_{\max }=15^{\circ}$.

\begin{tabular}{|c|c|c|c|c|c|}
\hline \multicolumn{3}{|c|}{$\begin{array}{c}\partial \phi_{\max }=5.25^{\circ} \text { corresponding to the } \\
\text { proposed tangent estimator }\end{array}$} & \multicolumn{5}{c|}{$\partial \phi_{\max }=15^{\circ}$ corresponding to the value used } \\
in [1]
\end{tabular}

Fig. 5. Impact of the proposed method on the error analysis [1] for Yuen's method [7]. The figures correspond to tests 4(a) and 4(b) of [1]. The dotted plot (red) shows the maximum error for the complete range of parameters, while the solid line (blue) shows the average error for the complete range for a particular value of $a \in[10,100]$. 


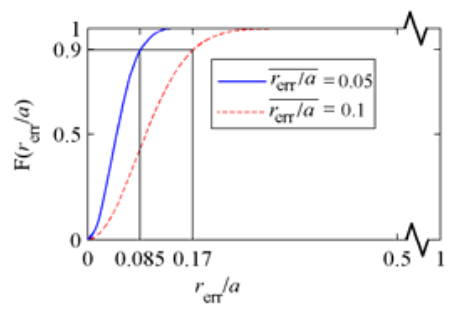

Fig. 6. The Rayleigh distribution function proposed in [1] for describing the probability density function of the relative error $\overline{r_{e r r} / a}$ in Yuen's method [7]. The distribution for the proposed tangent estimator uses $\overline{r_{\text {err }} / a}=0.05$, while $\overline{r_{\text {err }} / a}=0.1$ was used in [1].

The value of $\overline{r_{\text {err }} / a}=0.05$ obtained using the proposed tangent estimation method is very close to the $\overline{r_{e r r} / a}$ for the remaining tests in [1]. Thus, the error in estimation of tangents is no longer the most important contributing factor in the error analysis of [1]. In [1], the average value of relative error $\overline{r_{e r r} / a}$ is computed for four tests, and the maximum $\overline{r_{e r r} / a}$ among all the four tests is used to define probability density function (a Rayleigh's function) as below:

$$
\mathrm{F}\left(r_{\text {err }} / a\right)=1-\exp \left(-\left(r_{\text {err }} / a\right)^{2} /\left(2 \sigma^{2}\right)\right)
$$

where $\sigma=\overline{r_{\text {err }} / a} \sqrt{2 / \pi}$. The Rayleigh distributions obtained using $\overline{r_{\text {err }} / a}=0.05$ (corresponding to the proposed tangent estimation method, $\partial \phi_{\max }=5.25^{\circ}$ ) and $\overline{r_{\text {err }} / a}=0.1$ (originally used in [1] considering $\partial \phi_{\max }=15^{\circ}$ ) are shown in Figure 6.

In the methods, where Yuen's method [7] is used as a supplementary, or a part of the ellipse detection process, and the centers are computed using other methods (like least squares) as well, we can use (23) to choose a trust region. Suppose we want to verify the centers achieved using two methods, one of them is Yuen's method, then we may say that the computed centers using the two methods are $90 \%$ reliable if:

- For $\overline{r_{\text {err }} / a}=0.1$, the distance between the two centers is $r_{\text {err }} / a<=0.17$

- For $\overline{r_{\text {err }} / a}=0.05$, the distance between the two centers is $r_{\text {err }} / a<=0.085$

Thus, the proposed tangent estimation method provides a stricter trust region and the centers can be computed more reliably as well as precisely using the proposed tangent estimation method.

\section{Conclusion}

A simple, geometry based method is proposed for estimating the tangents of digital curves. The proof of the geometric concept used in the method is also presented. In 
addition, for digital ellipses, we perform the maximum error analysis and give explicit analytical terms for the maximum error. The maximum error in the tangent estimation using the proposed method is small (worst case $5.25^{\circ}$ for the considered examples).

It is shown that the proposed tangent estimation method can have significant impact on some practical applications. For example, it is shown that if good tangent estimation methods like the proposed method are used in a specific application, the trust region for computing the centers of the ellipses (using various methods) can be chosen more strictly, which shall enhance the reliability as well as the precision of the ellipse detection method.

At present, the analysis and results have been presented for digital ellipses and circles only. An example of digital curve with inflexion points is also considered. It is easily extensible to other conics like parabola, hyperbole, etc. It can also be extended to quadric curves (fourth order curves). Work is in progress for comparing the performance of the proposed tangent estimation method with the other tangent estimation methods. In this comparison, we shall consider various performance criteria like precision, maximal error, isotropy, convergence, convexity on ideal digital shape, and time complexity [12]. Such study shall help the image processing community in choosing good tangent estimators suitable for their corresponding applications while understanding the strengths and limitations of the tangent estimator used by them.

\section{References}

1. Prasad, D.K., Leung, M.K.H.: Error analysis of geometric ellipse detection methods due to quantization. In: Fourth Pacific-Rim Symposium on Image and Video Technology (PSIVT 2010), Singapore (2010)

2. Anderson, I.M., Bezdek, J.C.: Curvature and tangential deflection of discrete arcs: a theory based on the commutator of scatter matrix pairs and its application to vertex detection in planar shape data. IEEE Transactions on Pattern Analysis and Machine Intelligence PAMI 6, 27-40 (1984)

3. Coeurjolly, D., Klette, R.: A comparative evaluation of length estimators of digital curves. IEEE Transactions on Pattern Analysis and Machine Intelligence 26, 252-258 (2004)

4. Kovalevsky, V.: Curvature in digital 2D images. International Journal of Pattern Recognition and Artificial Intelligence 15, 1183-1200 (2001)

5. Lee, C.-K., Haralick, R.M., Deguchi, K.: Estimation of curvature from sampled noisy data. In: Proceedings of the IEEE Computer Society Conference on Computer Vision and Pattern Recognition, New York, USA, pp. 536-541 (1993)

6. Worring, M., Smeulders, A.W.M.: Digital curvature estimation. Computer Vision and Image Understanding 58, 366-382 (1993)

7. Yuen, H.K., Illingworth, J., Kittler, J.: Detecting partially occluded ellipses using the Hough transform. Image and Vision Computing 7, 31-37 (1989)

8. Feschet, F.: Canonical representations of discrete curves. Pattern Analysis and Applications 8, 84-94 (2005)

9. Faure, A., Buzer, L., Feschet, F.: Tangential cover for thick digital curves. Pattern Recognition 42, 2279-2287 (2009)

10. Tsai, D.M., Chen, M.F.: Curve fitting approach for tangent angle and curvature measurements. Pattern Recognition 27, 699-711 (1994) 
11. De Vieilleville, F., Lachaud, J.O.: Experimental comparison of continuous and discrete tangent estimators along digital curves. In: Brimkov, V.E., Barneva, R.P., Hauptman, H.A. (eds.) IWCIA 2008. LNCS, vol. 4958, pp. 26-37. Springer, Heidelberg (2008)

12. De Vieilleville, F., Lachaud, J.O.: Comparison and improvement of tangent estimators on digital curves. Pattern Recognition 42, 1693-1707 (2009)

13. Lewiner, T., Craizer, M.: Projective estimators for point/tangent representations of planar curves. In: 21st Brazilian Symposium on Computer Graphics and Image Processing, SIBGRAPI 2008, Campo Grande, BRAZIL, pp. 223-229 (2008)

14. Lewiner, T., Gomes Jr, J.D., Lopes, H., Craizer, M.: Curvature and torsion estimators based on parametric curve fitting. Computers and Graphics 29, 641-655 (2005)

15. Mokhtarian, F., Mackworth, A.: Scale-based description and recognition of planar curves and two-dimensional shapes. IEEE Transactions on Pattern Analysis and Machine Intelligence PAMI 8, 34-43 (1986)

16. Kerautret, B., Lachaud, J.O.: Robust estimation of curvature along digital contours with global optimization. In: Coeurjolly, D., Sivignon, I., Tougne, L., Dupont, F. (eds.) DGCI 2008. LNCS, vol. 4992, pp. 334-345. Springer, Heidelberg (2008)

17. Kim, E., Haseyama, M., Kitajima, H.: Fast and Robust Ellipse Extraction from Complicated Images. In: Proceedings of the International Conference on Information Technology and Applications, pp. 357-362 (2002) 